\title{
Control of Effluent Gases from Solid Waste Processing using Impregnated Carbon Nanotubes
}

\author{
Jing Li, John Fisher \\ (NASA Ames Research Center, Moffett Field, CA 94035) \\ Kanapathipillai Wignarajah \\ (Enterprise Advisory Services Inc, Ames Research Center, Moffett Field, CA 94035)
}

Copyright $(20) 5$ SAE International

\begin{abstract}
One of the major problems associated with solid waste processing technologies is effluent contaminants that are released in gaseous forms from the processes. This is a concern in both biological as well as physicochemical solid waste processing. Carbon dioxide $\left(\mathrm{CO}_{2}\right)$, the major gas released, does not present a serious problem and there are currently in place a number of flight-qualified technologies for $\mathrm{CO}_{2}$ removal. However, a number of other gases, in particular $\mathrm{NO}_{x}, \mathrm{SO}_{2}, \mathrm{NH}_{3}$, and various hydrocarbons (e.g. $\mathrm{CH}_{4}$ ) do present health hazards to the crew members in space habitats.

In the present configuration of solid waste processing in the International Space Station (ISS), some of these gases are removed by the Trace Contaminant Control System (TCCS), demands a major resupply. Reduction of the resupply can be effective by using catalyst impregnated carbon nanotubes. For example, NO decomposition to $\mathrm{N}_{2}$ and $\mathrm{O}_{2}$ is thermodynamically favored. Data showing decomposition of NO on metal impregnated carbon nanotubes is presented. Comparisons are made of the existing TCCS systems with the carbon nanotube based technology for removing $\mathrm{NO}_{x}$ based on mass/energy penalties.
\end{abstract}

\section{INTRODUCTION}

Processing of solid wastes invariably requires the use of thermal methods in order to destroy potentially harmful biological organisms and organic compounds. A major problem encountered during such processes is the release of decomposition products in the form of gases that can be harmful to humans. Other sources are off gases coming from structural materials of space vehicle, crew activities (e.g. exercise, food preparation etc), experimental payload operations, accidental releases, microbial metabolism and contingency events. An excellent compilation of the list of chemicals released into the space cabin is provided in references $[1,2]$.

We present our recent investigation of using SWNTs as catalytic supporting materials to impregnate metals, such as rhodium (Rh), potassium $(K)$ and other catalysts. A protocol has been developed to impregnate the $\mathrm{Rh}$ and $\mathrm{K}$ in aqueous metal chloride solution, according to unique surface properties of SWNTs. The Rh has been successfully impregnated in SWNTs. The SWNTs, Rh-SWNTs and K-SWNTs have been characterized by thermogravimetric analysis (TGA) and scanning electron microscope (SEM). The NO decomposition is characterized by a residual gas analyzer (RGA).

The project is funded by a NASA Research Announcement Grant to find applications of single walled carbon nanotubes in eliminating toxic gas contaminant in a life support system. This knowledge will be utilized in the development of a prototype SWNT $\mathrm{NO}_{x}$ gas purification system that would represent a significant step in the development of high efficiency systems capable of selectively removing specific gaseous for use in regenerative life support systems for human exploration missions.

\section{THEORY OF NO REDUCTION}

The theory of NO reduction on metals has been extensively studied. Rhodium metal appears to be one of the best catalyst for this purpose [3]. The key step of the NO reduction has long been thought to be the breaking of the O-bond upon chemisorption onto the metal. The bond energy and bond length are 149.9 $\mathrm{Kcal} / \mathrm{mol}$ and $1.2 \mathrm{~A}$. NO is thermodynamically unstable $\left(\Delta \mathrm{G}_{298}^{0}=86.32 \mathrm{KJ} / \mathrm{mol}\right)$ but once formed it's decomposition at 1 Atmosphere is kinetically notfavored, though it can be broken down at higher pressures [4]. Rapid recombination of surface nitrogen 
atoms to form $\mathrm{N}_{2}$ follows rapidly. The remaining oxygen atom then is released as $\mathrm{O}_{2}, \mathrm{CO}$ or $\mathrm{CO}_{2}$ depending on the conditions and the supporting matrix of the catalyst. Luo et al. [5] demonstrated the decomposition of NO on carbon nanotubes and rhodium impregnated carbon nanotubes. Since carbon nanotubes are relatively nonreactive, we believe that the atomic oxygen will be released as oxygen gas.

Alkali metals are known to act as catalysts in carbon gasification reactions. Kapteijn et al. [6] observed a large increase in NO reduction (and carbon reactivity) when $\mathrm{K}$ was added to the carbon. Okukara and Tananka [7] found that, upon potassium addition to activated carbon, both the NO adsorption capacity and carbon reactivity increased. Illan-Gomez et al. $[8,9]$ studied NO reduction by $\mathrm{K}$-loaded activated carbon in detail and found that the catalytic role of $\mathrm{K}$ was attributed to its effective participation in an oxidation-reduction (redox) cycle between $\mathrm{K}_{x} \mathrm{O}_{\mathrm{y}}$ and $\mathrm{K}_{\mathrm{x}} \mathrm{O}_{\mathrm{y}+1}$, in which the catalyst is oxidized by $\mathrm{NO}$ and reduced by carbon to produce $\mathrm{N}_{2}$ and $\mathrm{CO}_{2}$.

\section{MATERIALS AND METHODS}

\section{SOURCES OF CARBON NANOTUBES}

The purified single walled carbon nanotubes containing less than $2 \%$ impurities in this study were purchased from Carbon Nanotechnolgies Inc, Houston, TX. The method of producing the SWNTs is by the high-pressure carbon monoxide disproportionation (HiPCo) reaction [9]. This raw HiPCo SWNTs normally contains between $22-30 \%$ iron originating from the catalyst used for carbon nanotube growth. These samples obtained from Rice University are referred to as "Raw HiPCo" in the paper.

\section{RHODIUM CATALYST PREPARATION}

Rhodium impregnation was achieved by adding a known concentration of rhodium chloride solution to a preweighed sample of carbon nanotubes. The rhodium chloride solution is prepared so that when added to the carbon nanotubes, the percentage of metallic rhodium ranged from $1-20 \%$. A mild vacuum was applied for 1 hour and the sample stirred overnight. The mixed solution was dried for $8-12$ hours at $105^{\circ} \mathrm{C}$ in an atmosphere of $\mathrm{N}_{2}$. The dried sample was reduced in a stream of $10-20 \% \mathrm{H}_{2}$ in Argon with heating from room temperature to $200{ }^{\circ} \mathrm{C}$ at a temperature ramp of $5^{\circ} \mathrm{C}$ per minute. When the temperature reached $200{ }^{\circ} \mathrm{C}$, the sample was maintained in the $\mathrm{H}_{2} / \mathrm{Ar}$ atmosphere for a further 30 minutes. TGA analysis, SEM imaging was done on the samples prior to testing its catalytic activity.

\section{POTASSIUM CATALYST PREPARATION}

Potassium carbon nanotube catalysts were prepared by adding a known concentration of "Analytical Grade" potassium nitrate to a pre-weighed sample of carbon nanotubes with the concentration being so designed to produce a $20 \% \mathrm{~K}$ carbon nanotube catalyst. The mixture was stirred for 24 hours and then dried at $105^{\circ} \mathrm{C}$ overnight. The sample was subjected to a 1-hour treatment in helium at $300^{\circ} \mathrm{C}$ to decompose any adsorbed nitrate. TGA analysis and SEM imaging was done on the samples before using it for catalytic activity.

\section{CATALYCTIC ACTIVITY MEASUREMENT}

A concentration of $250 \mathrm{ppm}$ NO in helium was used as the source of NO. The choice of the concentration was based on two major criterion namely the known concentrations of $\mathrm{NO}_{x}$ in typical flue gases and the sensitivity of analysis using the residual gas analyzer (RGA). The basic protocol involved placing an accurately weighed amount of the metal catalyst between 35-100 $\mathrm{mg}$ into the sample tube. Glass wool was placed on top and below the sample to ensure that the sample is held firmly in the tube during the experiment.

The catalyst was first treated with $\mathrm{H}_{2}(99.9 \%)$ gas to ensure that the rhodium remained in the metallic form followed by a flow of $\mathrm{He}(99.999 \%)$ to flush out residual $\mathrm{H}_{2}$ and measure the free space. Subsequently, the 250 ppm NO in He was passed through the catalyst-SWNTs in the sample tube at different temperatures temperatures of $200,250,300^{\circ} \mathrm{C}$ for 20 minutes of each in the case of the Rh-SWNTs and temperatures of 150 , $200,250,275$ and $300^{\circ} \mathrm{C}$ for the K-SWNTs. A temperature of $400^{\circ} \mathrm{C}$ was used only once with the $20 \%$ rhodium-SWNTs and subsequently it was decided not to use this temperature as we noticed evolution of $\mathrm{CO}_{2}$ that suggested that it was possible that some of carbon matrix was being oxidized. The gas flow of $\mathrm{H}_{2}, \mathrm{He}$ and $\mathrm{NO}$ was controlled by a MKS 640A Absolute Pressure Controller for closed-loop electronic pressure control. The pressure at the outlet of sample tube was accurately controlled at 761 Torr to ensure a gas flow through the tube, see a flow chart in Figure 1.

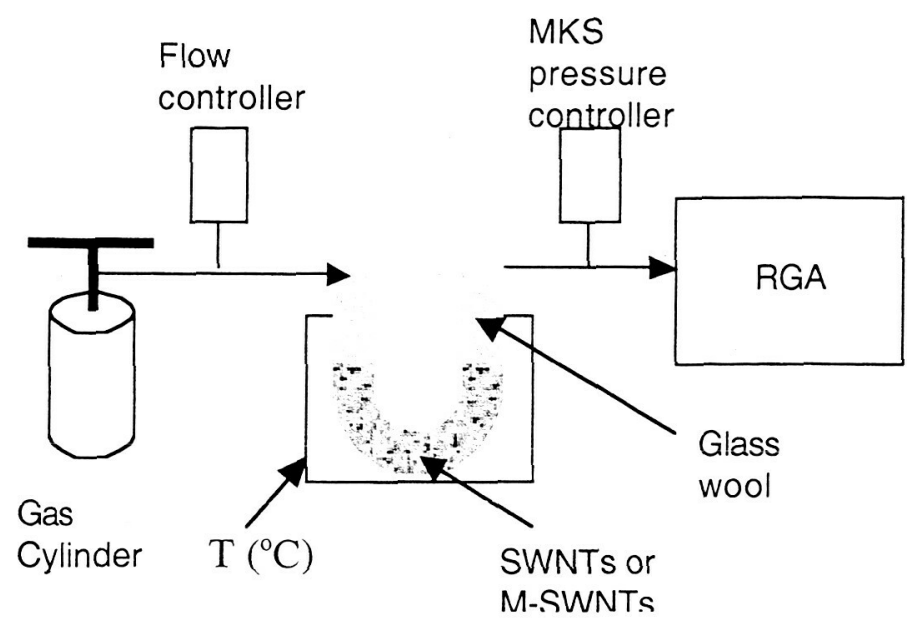

Figure 1 A flow diagram of the setup for NO conversion measurement. 
The NO conversion measurement protocol is summarized in table 1 below. There are slightly difference in timing for the blank tube control experiment and Rh-SWNT, K-SW'Nivis samples.

Table 1: Protocol for gas flow, temperature and reaction time in Chemisorption studies.

\begin{tabular}{|c|c|c|c|}
\hline PROCESS & TEMP $\left({ }^{\circ} \mathrm{C}\right)$ & $\begin{array}{l}\text { RAMP } \\
\operatorname{RATE}\left({ }^{\circ} \mathrm{C} / \mathrm{min}\right)\end{array}$ & TIME (min) \\
\hline Evacuate & 150 & 10 & 20 \\
\hline Flow H2 & 150 & 10 & 20 \\
\hline Evacuate & 150 & 10 & 20 \\
\hline Flow $\mathrm{He}$ & 150 & 10 & 20 \\
\hline Evacuate & 150 & 10 & 20 \\
\hline $\begin{array}{l}\text { Flow NO or } \\
\mathrm{He}\end{array}$ & 150 & 5 & $20-30$ \\
\hline Evacuate & 200 & 10 & 20 \\
\hline $\begin{array}{l}\text { Flow NO or } \\
\mathrm{He}\end{array}$ & 200 & 5 & $20-30$ \\
\hline Evacuate & 250 & 10 & 20 \\
\hline $\begin{array}{l}\text { Flow NO or } \\
\mathrm{He}\end{array}$ & 250 & 5 & $20-30$ \\
\hline Evacuate & 250 & 10 & 20 \\
\hline $\begin{array}{l}\text { Flow NO or } \\
\mathrm{He}\end{array}$ & 300 & 5 & $20-30$ \\
\hline Evacuate & 300 & 10 & 20 \\
\hline
\end{tabular}

\section{THERMOGRAVIMETRIC ANALYSIS}

A Perkin Elmer thermogravimetric analyzer (TGA Pris 1) was used to measure the metal contents in the SWNTs, Rh-SWNTs and K-SWNTs samples by burning off all carbonaceous material and check the percentage of residules. The carrier gas was nitrogen with a flow rate of $20 \mathrm{cc} / \mathrm{min}$. The temperature ramping rate was set at $10^{\circ} \mathrm{C} / \mathrm{min}$. The temperature range was set from $50^{\circ} \mathrm{C}$ to $1025^{\circ} \mathrm{C}$

\section{SCANNING ELECTRON MICROSCOPE}

The carbon nanotube impregnated with rhodium was dispersed in dimethyformamide (DMF). This solution was drop deposited onto a silicon dioxide substrate. After the DMF evaporated the Rh-SWNTs lay on the silicon dioxide for SEM imaging.

\section{RESULTS}

\section{SEM IMAGE OF RH-SWNTS}

The SEM image of Rh-SWNTs sample is displayed in Figure 2. The rhodium forms a type of flower shape nestled with carbon nanotube bundles. The size of carbon nanotubes bundles is about $20-30 \mathrm{~nm}$ and the rhodium flower is in the diameter of $500 \mathrm{~nm}$. This flower shape of rhodium is reproducible from spot to spot with similar size. They may be formed during the drying of $\mathrm{RhCl}_{2}$-SWNTs from water while the rhodium was still in the form of rhodium chloride salt. After the hydrogen reduction of rhodium in dry phase of Rh-SWNTs, the rhodium spots may stay in the shape as they were in the form of salt. The preference of $\mathrm{Rh}$ shaped in carbon nanotubes needs more exploitation and the size of $R h$ cluster will be studied for them to be controllable with correlation to the NO conversion efficiency.

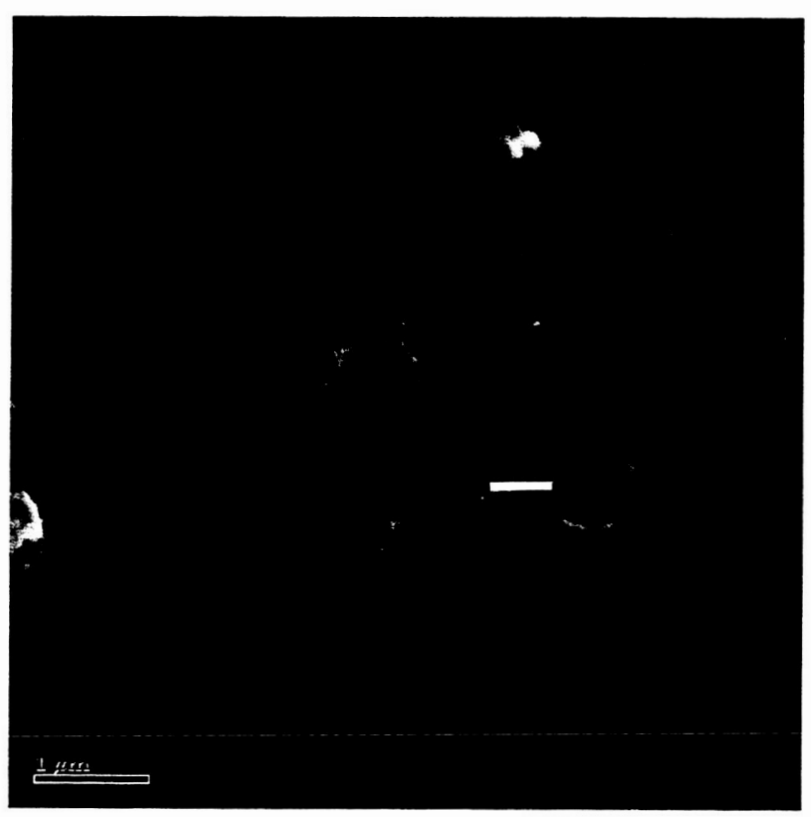

Figure 2 SEM image of rhodium impregnated single wall carbon nanotubes

\section{THERMOGRAVIMETRIC ANALYSIS DATA}

Figure 3 shows a thermogravimetric analysis of the different materials with nitrogen as a carrier gas. The nonimpregnated SWNTs begins to decompose at temperatures between $375-400^{\circ} \mathrm{C}$ and plateaus at around $1.6 \%$ confirming that the carbon nanotubes do have less than $2 \%$ impurities as reported by the supplier. In the presence of rhodium, decomposition of the carbon nanotubes begins at a lower temperature around $325-350^{\circ} \mathrm{C}$, while in the presence of potassium it begins at temperatures even lower below $300^{\circ} \mathrm{C}$. The rhodium 
content of one catalyst sample had $18 \%$ and the second $15 \%$ based on the TGA analysis. The potassium catalyst

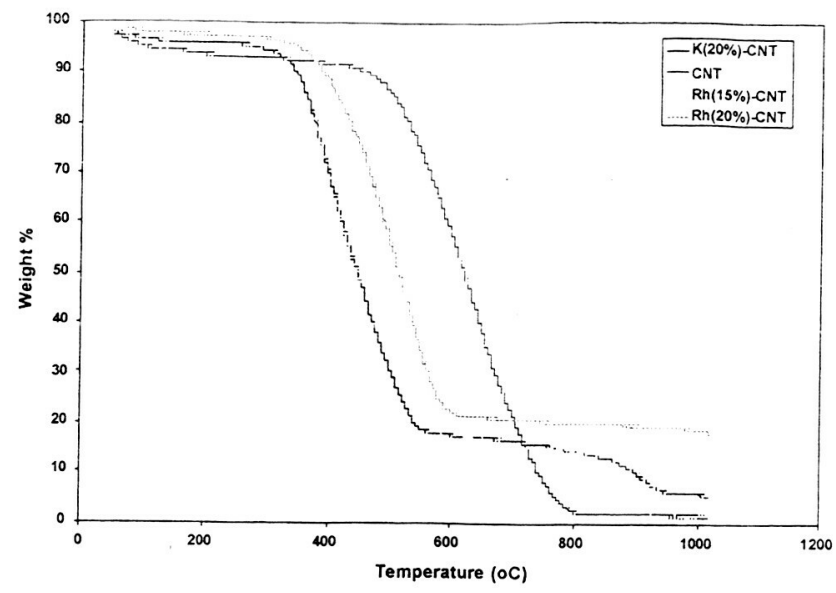

Figure 3 TGA plots of different materials: SWNTs, Rh(15\%)-SWNTs, Rh(18\%)-SWNTs, K(19\%)-SWNTs

had a potassium content of around $19 \%$. These results enabled us to make a decision on the temperature ranges that were considered suitable for performing catalytic studies without burning off the carbon nanotubes matrix.

\section{EFFECT OF TEMPERATURE ON NO REDUCTION}

Reduction of NO was carried out in the Micromeritics 2010 instrument using the chemisorption setup that the temperature can be ramped above room temperature.

A preliminary study with $20 \%$ Rhodium impregnated carbon annotates suggested that at temperatures around $350-400^{\circ} \mathrm{C}$, the carbon in the catalyst was being oxidized as evidence by the evolution of $\mathrm{CO}_{2}$ detected by RGA. In order to eliminate the oxidation of carbon nanotubes by $\mathrm{NO}$ gas, the operating temperature for NO reduction was then setup equal or below $300^{\circ} \mathrm{C}$ for rest of the study in this paper.

Figure 4a shows a RGA scan data for 250ppm NO injection into a sample tube for a control (Blank tube). It is clear that the NO peak kept a constant value over the temperature of $200,250,300^{\circ} \mathrm{C}$. There is no readout from $\mathrm{CO}_{2}$ peak at these temperatures except a cosmos spike occurred when NO sample was first time injected. The control experiment tells the levels of NO peak and $\mathrm{CO}_{2}$ peak without possible conversion taking place.

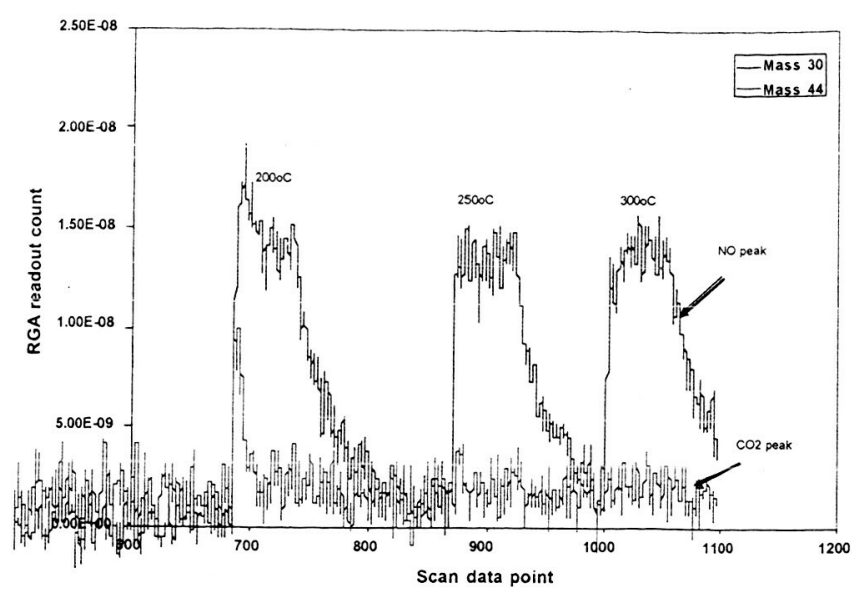

Figure 4a RGA scan data from the control experiment (blank sample tube).

Figure $4 \mathrm{~b}$ shows a RGA scan data for 250ppm NO injection into a sample tube contained $15 \%$ rhodium impregnated SWNTs at temperatures of 200,250 and $300^{\circ} \mathrm{C}$. At the lower temperatures of 200 and $250^{\circ} \mathrm{C}$, the RGA readout level of $\mathrm{NO}$ is not significantly smaller than that in the control experiment. This means very minimal conversion taking place at these temperatures (200 and $250^{\circ} \mathrm{C}$. However, NO decomposition occurred at $300^{\circ} \mathrm{C}$ virtually no NO was detected after the No was passed through the catalyst. Compared with the results from the control experiment, it would suggest that the Rh-SWNTs driven NO reduction is almost $100 \%$ complete at $300^{\circ} \mathrm{C}$. The cause of NO reduction can be considered in two possible ways; 1) NO decomposed on the surface of rhodium, 2) NO oxidized the carbon nanotubes to form $\mathrm{CO}_{2}$. There are spikes showing in $\mathrm{CO}_{2}$ peak in Figure $4 \mathrm{~b}$. However, the spikes are so sharp that could be the cosmos pulse or due the valve switch. And the $\mathrm{CO}_{2}$ level is down towards the baseline after the spikes, which means the $\mathrm{CO}_{2}$ was not formed in a significant amount, if there is any, to be maintained at a constant level. This may indicate that the NO reduction is mainly caused by catalysis of Rh in the SWNTs.

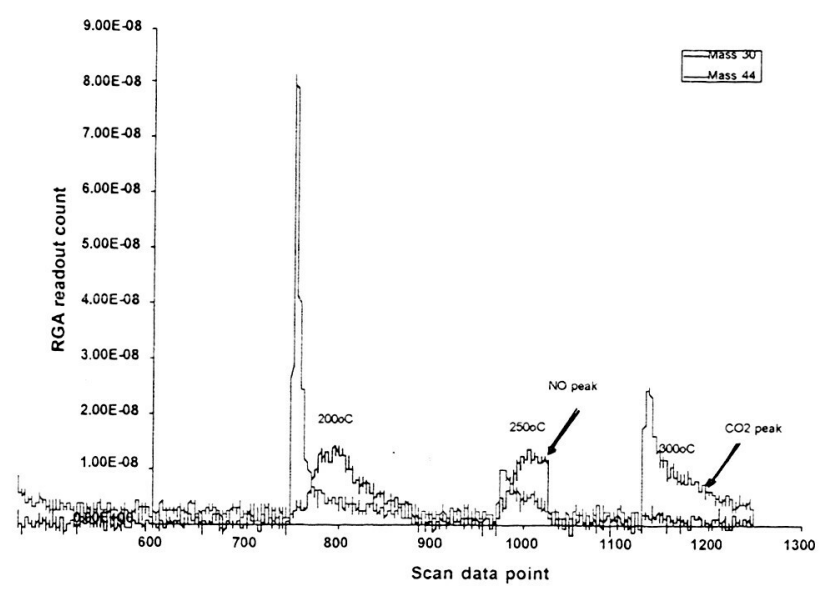


Figure 4b RGA scan data from Rh-SWNTs sample for NO conversion measurement

are currently in progress to determine the temperature optimum specifically between 250 and $300^{\circ} \mathrm{C}$.

Further studies are also in progress to study the optimal metal concentration required for NO decomposition. Luo using 1\% Rh-SWNTs reported only about about $10 \%$ NO decomposition at 300 oC. It is critical for NASA's application that the activity be expressed as a function of concentration of NO, since rates of generation of NO on ISS are well known and the permissible SMAC levels are established.

\section{ACTIVITY OF POTASSIUM IMPREGNATED CARBON ANAOTUBES}

Decomposition of NO at low temperatures has been reported by llan-Gomez et al (1995). We have measured the out gases after the NO passing through the $\mathrm{K}$ SWNTs at $150,200,250,275$, and $300^{\circ} \mathrm{C}$. There is no obvious reduction of NO observed. The NO peak level was maintained a constant at all these temperatures and similar to the level in the control experiment.

\section{POWER/MASS PENALTIES \& ESM}

On the Russian Space Station, Mir, the trace contaminant control system was composed of 5 components - a fan, an expendable charcoal canister weighing $6 \mathrm{Kg}$ and 2 regenerable charcoal canisters weighing $23.4 \mathrm{Kg}$ each. The regenerable canisters were regenerated every 20 days by exposing to space vacuum for 60 minutes followed by heating to between 180 and $200^{\circ} \mathrm{C}$ for 1.5 hours. The system processes 20 $\mathrm{m}^{3}$ of air/h and handles a number of contaminants. The nitrogen oxide generation rate is around $13.5 \mathrm{mg} /$ day and the trace control system maintains the nitrogen oxides within the SMAC value. $0.3 \mathrm{mg} / \mathrm{m}^{3}$. This same system is in place on ISS but recently a thermal oxidation was added in which a $\mathrm{Pd} / \mathrm{Pt}$ catalyst on Alumina was added. Unlike the United States TCCS system, the Russian system operates at a lower temperature (200$250^{\circ} \mathrm{C}$ ) and this is achieved by flowing at a lower flow rate of $0.5 \mathrm{~cm}^{3} /$ hour. Other major differences between the Russian and US TCCS system include (a) the Russian air handling capacity is greater and in the present configuration in ISS has been increased to handle 27 $\mathrm{m}^{3} /$ hour vs $15.3 \mathrm{~m}^{3} /$ hour (b) The Russian system purifies almost $37,000 \mathrm{Kg}$ of air per $1.28 \mathrm{KW}$.hr, while the original US design was only capable processing $928 \mathrm{Kg}$ of air per 7.63 KW.hr. Clearly, the Russian system was more efficient from an energy perspective. The Rh-carbon nanotube catalyst will operate at lower temperatures than the existing catalyts system $\left(275-300^{\circ} \mathrm{C}\right.$ vs $\left.400{ }^{\circ} \mathrm{C}\right)$. Preliminary estimate assumes a slow flow rate of $1.7 \mathrm{~m}^{3} / \mathrm{hr}$ contaminated air flow through the catalyst. With this slow flow rate, it is estimated that $0.06 \mathrm{KW}$.hr energy will be required to destroy the $13.5 \mathrm{mg}$ of NO produced per day by a 6 person crew.

\section{CONCLUSION}

The 7-day Space Craft Maximum Allowable Contaminant (SMAC) level for $\mathrm{NO}, \mathrm{NO}_{2}$ and $\mathrm{N}_{2} \mathrm{O}$ are in ppm 4.9, 0.5 and 491 and in $\mathrm{mg} / \mathrm{m}^{3} 6.1,0.94$ and 900 respectively. In order to ensure the safety of the crew, it is essential that these standards be met.

TGA analysis of the prepared catalytic materials showed that rhodium metal and the potassium metal contents in the SWNTs matrix are within experimentally acceptable levels of the required metal/ion ratio.

SEM images of the Rh-SWNTs showed that the rhodium appeared to be in clusters nestled in the bulk of the carbon nanotubes bundle meshes.

We have provided evidence that $250 \mathrm{ppm}$ NO can be completely decomposed with a $15 \%$ Rh-SWNTs at $300^{\circ} \mathrm{C}$. There is no reduction of NO from the $19 \% \mathrm{~K}$ SWNTs up to $300^{\circ} \mathrm{C}$.

\section{ACKNOWLEDGMENTS}

Authors would like to thank Mark Anderson, Kevin Howard, Martin Cinke and Brett Cruden for excellent technical and laboratory assistance. This project is supported by NASA Research Anouncement award

\section{REFERENCES}

1. J.L. Perry Elements of Spacecraft cabin Air Quality Control Design NASATP 207978, Marshall Space Flight Center, AL (1998).

2. T.Wydeven and M.A. Golub Generation rates and chemical composition of waste streams in a typical crewed space habitat. NASA Tech. Memo. 102799, NASA-Ames Research Center, CA (1990).

3. K.C.Taylor, Nitric oxide catalysts in automotive exhaust systems. Catal. Rev. Sci. \& Eng. 35:457 (1993)

4. J.S.Beckman The physiological and pathological chemistry of nitric oxide. In. Lancaster.J, (ed). Nitric Oxide - Principles and Actions. New York: Academic Press; pp 1-83 (1996)

5. J.Z.Luo, L.Z Gao, Y.L. Leung and C.T.Au The decomposition of NO on CNT's and $1 \%$ wt $\%$ Rh/CNT's Catalysis Letters 66:91-97 (2000)

6. F, Kapteijn, J.C. Alexander, G.A. Mierop, and J.A. Moulijn, J. Chem. Soc. Chem. Commun. 1084 (1984)

7. T. Okukara, K Tanaka, J. Chem. Soc. Faraday Trans 82: 3657 (1986) 
8. M.J.llan Gomez, A. Linares-Solano, L.R. Radovic, C. Salinas-Martinez de Lecea. NO reduction by activated carbons. 2. Catalytic Effect of Potassium. Energy and Fueis 9:97-103 (1995)

9. M.J. Illan-Gomez, ALinares-Solano, L.R. Radovic, C. Salinas-Martinez de Lecea NO reduction by activated carbons. 7. Some mechanistic aspects of uncatalyzed and catalyzed reaction Energy Fuels 10: 158 (1996)

\section{CONTACT}

Jing Li- E-mail: jingli@mail.arc.nasa.gov; Phone 1-650604-4352
DEFINITIONS, ACRONYMS, ABBREVIATIONS

CNT Carbon Nanotubes

HiPCo High Pressure Carbon monoxide disproportionation reaction

Rh-SWNTs Rhodium impregnated single wall carbon nanotubes

K-SWNTs Potassium impregnated single wall carbon nanotubes

SEM Scanning Electron Microscopy

SMAC Space Station Maximum Allowable Contaminant levels

TGA Thermogravimetric Analysis

RGA Residual gas analyzer 